\title{
Propiverine-induced accumulation of nuclear and cytosolic protein in F344 rat kidneys: Isolation and identification of the accumulating protein
}

\author{
D.R. Dietrich ${ }^{a, *, 1}$, A.H. Heussner ${ }^{a, 1}$, E. O'Brien ${ }^{a, 1}$, T. Gramatté ${ }^{b, 2}$, M. Runkel ${ }^{b}$, S. Rumpf ${ }^{c}$, B,W. Day ${ }^{\text {d,e }}$ \\ a Environmental Toxicology, University of Konstanz, 78457 Konstanz, Germany \\ ${ }^{b}$ Apogepha Arzneimittel GmbH, 01281 Dresden, Germany \\ ᄃ IPMC-TMC GmbH, 8566 Neuwilen, Switzerland \\ ¿ Department of Pharmaceutical Sciences, University of Pittsburgh, Pittsburgh, PA 15213, USA \\ \& Department of Chemistry, University of Pittsburgh, Pittsburgh, PA 15213, USA
}

\section{A R T I C L E I N F O}

\section{Article history:}

Received 25 March 2008

Revised 10 September 2008

Accepted 11 September 2008

Available online 27 September 2008

\section{Keywords:}

Propiverine

d-Amino acid oxidase

F344 rats

LPMC

Immunohistochemistry

MALDI-TOF-MS

\begin{abstract}
A B S T R A C T
Male and female F344 rats but not B6C3F1 mice exposed for 104 weeks to propiverine hydrochloride (1-methylpiperid-4-yl 2,2-diphenyl-2-(1-propoxy)acetate hydrochloride), used for treatment of patients with neurogenic detrusor overactivity (NDO) and overactive bladder (OAB), presented with an accumulation of proteins in the cytosol and nuclei of renal proximal tubule epithelial cells, yet despite this, no increased renal tumor incidence was observed. In order to provide an improved interpretation of these findings and a better basis for human health risk assessment, male and female F344 rats were exposed for 16 weeks to 1000 ppm propiverine in the diet, the accumulating protein was isolated from the kidneys via cytosolic and nuclear preparations or laser-capture microdissection and analyzed using molecular weight determination and mass spectrometry. The accumulating protein was found to be D-amino acid oxidase (DAAO), an enzyme involved in amino and fatty acid metabolism. Subsequent reanalysis of kidney homogenate and nuclear samples as well as tissue sections using western blot and DAAO-immunohistochemistry, confirmed the presence and localization of DAAO in propiverine-treated male and female F344 rats. The accumulation of DAAO only in rats, and the limited similarity of rat DAAO with other species, including humans, suggests a rat-specific mechanism underlying the drug-induced renal DAAO accumulation with little relevance for patients chronically treated with propiverine.
\end{abstract}

\section{Introduction}

The benzilic acid derivative propiverine hydrochloride (1-methylpiperid-4-yl 2,2-diphenyl-2-(1-propoxy)acetate hydrochloride) was developed by Apogepha Arzneimittel $\mathrm{GmbH}$, Dresden (Germany) and first registered as the immediate release drug formulation Mictonorm $^{\circledast}$ in the German Democratic Republic (GDR) in 1981 (May et al., in press). Due to its neurotropic and musculotropic effects on the urinary smooth muscle, propiverine is used for treatment of patients with neurogenic detrusor overactivity (NDO) and overactive bladder $(\mathrm{OAB}$, frequency and urge with/without urge incontinence)(Madersbacher, 2006). Upon oral administration, propiverine is rapidly and nearly completely absorbed and undergoes extensive and rapid firstpass metabolism in the liver to the main metabolite propiverine $\mathrm{N}$ -

\footnotetext{
* Corresponding author. Human and Environmental Toxicology, University of Konstanz, PO-Box: X-918, 78457 Konstanz, Germany. Fax: +497531883170. E-mail address: daniel.dietrich@uni-konstanz.de (D.R. Dietrich).

1 These authors contributed equally to the publication.

2 Current address: Drug Development Consulting, Schönstrasse 73, 81543 Munich, Germany.
}

oxide (Fig. 1). Due to high plasma protein binding of both the parent compound (ca. 90\%) and the N-oxide metabolite (ca. 60\%), both demonstrate low renal clearance of $<1 \mathrm{ml} / \mathrm{min}$ (Madersbacher and Mürtz, 2001). Despite this, drug accumulation does not occur following repeated and long-term treatment, but rather a steady state mean trough level of approx. $60 \mathrm{ng} / \mathrm{ml}$ plasma is observed when healthy volunteers are repeatedly given therapeutically effective doses of $15 \mathrm{mg}$ t.i.d or $45 \mathrm{mg}$ s.i.d (May et al., 2008).

Despite approval and marketing authorization in the GDR and subsequent treatment of patients, propiverine was subjected to preclinical and clinical testing as of 1989 in order to attain drug approval for marketing in Japan and later in additional European countries via the mutual recognition procedure (EMEA, 2003). A 52-week oral study with rats (Yamashita et al., 1990) and a 104-week oral carcinogenicity bioassay with rats and mice (Inoue et al., 1989a; Inoue et al., 1989b) revealed interesting effects in that clear sex differences in the degree of pathology (non-neoplastic, pre-neoplastic and neoplastic lesions) were observed in male and female F344 rat kidneys while no renal

\footnotetext{
${ }^{3}$ The two reports (Apogepha report No,P-148 and $\mathrm{P}-150$ ) can be obtained per direct request from Apogepha GmbH in Dresden, Germany (www.apogepha,de).
} 


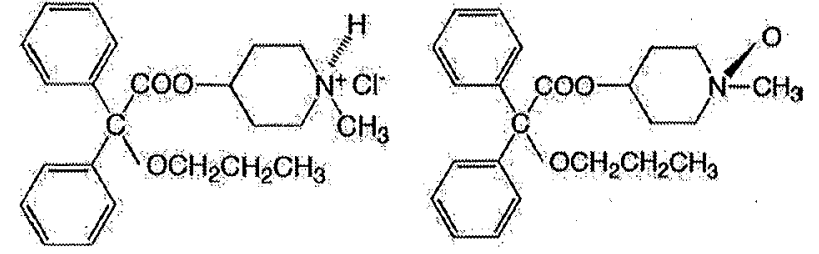

Fig. 1. Structures of propiverine and its major metabolite propiverine- $\mathrm{N}$-oxide (Wuest et al., 2005).

pathological changes were observed in either sex of the B6C3F1 mice F344 rat renal pathology consisted primarily of prominent hyaline droplets in the cytosol and nuclei of proximal tubule epithelial cells in both sexes. Pathologies in the renal cortex, medulla and papilla, were decisively more pronounced in males than in females. Similarly, increased incidences of simple hyperplasia were observed in the transitional epithelium of the papilla of male but not female rats with the highest incidences noted at the highest dose (1000 ppm) of propiverine applied (Inoue et al., 1989a).

In contrast, a one-year study with beagle dogs did not reveal any pathological changes in the kidneys (Kohda et al., 1989) thus raising the question whether the more apparent renal pathology observed in male than in female F344 rats is species-specific with a sex-specific component. Similar hyaline droplets in the nuclei of rat proximal tubule epithelial cells have previously been described (Gopinath et al, 1988). However, neither the treatment regimen nor the compound used in this study was disclosed and the accumulating protein(s) was not isolated, characterized or identified.

One of the best-characterized pathologies associated with renal hyaline droplet accumulation pathology is the male rat-specific $\alpha 2 \mathrm{u}$ globulin nephropathy and carcinogenicity (Swenberg, 1991; Dietrich, 1995). As $\alpha 2 u$-globulin nephropathy is observed as cytosolic but not nuclear protein accumulation in proximal tubule epithelial cells of male rats only (females do not synthesize the hepatic isoform of $\alpha 2 u$ globulin) (Dietrich and Rasonyi, 1995), the propiverine-induced accumulation of hyaline droplets in the renal proximal tubules of both male and female rats following propiverine exposure is unlikely to consist of $\alpha 2 \mathrm{u}$-globulin. More likely is that these hyaline droplets represent several proteins accumulating concurrently, with a contribution of $\alpha 2 \mathrm{u}$-globulin to the hyaline droplet pathology in male but not in female rats, thus possibly explaining the sex-specific component in the observed more prominent renal pathology in male F344 rats. As a first step towards providing a better understanding of this hyaline droplet phenomenon and its importance for human cancer risk assessment, and in order to distinguish it from the known $\alpha 2 \mathrm{u}$ globulin nephropathy, the protein(s) involved in the propiverineinduced hyaline droplet formation was isolated and identified.

\section{Materials and methods}

Chemicals and reagents. Unless otherwise stated, all chemicals were purchased from Sigma-Aldrich $\mathrm{GmbH}$, Germany. Rat diet pellets, placebo and 1000 ppm propiverine-supplemented, were prepared by Ssniff Spezialdiäten GmbH, Germany.

Animals, exposure regimen, and sample collection. Forty male and 40 female F344 rats (Charles River Laboratories GmbH, Germany) of 120$140 \mathrm{~g}$ body weight were housed at the University of Konstanz animal research facility (TFA). They were randomly marked by ear piercing, and allocated to cages in groups of five for acclimatization 26 days prior to study start. Animal housing and experimentation were approved by the animal care and use committee of the university of Konstanz and subject to permission by the responsible authority (Regierungspräsidium Freiburg, Germany).
Thirty male and 30 female F344 rats were placed on a 1000 ppm propiverine-supplemented diet for 16 weeks. The control group (10 males and 10 females) received placebo feed (see supplemental information, Figure S1). Feeding was ad libitum and food and water consumption per cage were monitored weekly. Animals were examined daily and weighed weekly.

At termination of exposure, animals were anaesthetized with pentobarbital sodium ( $50 \mathrm{mg} / \mathrm{ml}, 2.5 \mathrm{ml} / \mathrm{kg}$ i.p.). A total of five animals per treatment group and sex were retrograde perfused (Dietrich and Swenberg, 1991a; Dietrich and Swenberg, 1991b; Dietrich and Swenberg, 1991c). Right kidneys were sliced sagitally (three sections) and placed in histology fixative (2\% (w/v) paraformaldehyde and $1 \%$ glutaraldehyde in $82.4 \mathrm{mM} \mathrm{NaH} 2 \mathrm{PO} 4$ adjusted with $66.2 \mathrm{mM} \mathrm{NaOH}$ to pH $7.6(864 \mathrm{mOsM})$ ), embedded in paraffin, sectioned $(2-3 \mu \mathrm{M})$ and stained with Mayer's hematoxylin and eosin (H\&E, RCC Ltd., Switzerland). Left kidneys and livers were removed, snap-frozen in liquid nitrogen and stored at $-80^{\circ} \mathrm{C}$ for subsequent analyses. Organs (nonperfused) from the remaining animals per treatment group were taken for protein analysis (see supplemental information, Figure S1).

Pathological reading of the kidney sections. Hematoxylin and eosin ( $H \& E$ ) stained kidney sections of five animals per treatment group and sex were randomized for subsequent double-blind pathological assessment. Selected pathological lesions were photographed using a Nikon CoolPix950 digital camera mounted on a Zeiss microscope.

Renal cortex and liver protein extraction, concentration determination, SDS-PAGE and silver staining. Nuclear and cytosolic proteins were extracted from kidney (pooled cortices of five animals) and liver samples according to published protocols (Blobel and Potter, 1966). Protein concentrations of extracts were determined at $\lambda=595 \mathrm{~nm}$ using a bovine $\gamma$-globulin standard calibration curve according to Bradford (Bradford, 1976). Samples were diluted with electrophoresis sample buffer to a final protein concentration of $2 \mu \mathrm{g} / \mu \mathrm{l}$ (Laemmli, 1970 ) and a total of $20 \mu \mathrm{g}$ protein per lane was applied to $12 \%$ polyacrylamide gels. Analytical gel silver staining and molecular weight was estimated according to published methods (Heukeshoven and Dernik, 1985). For samples destined for mass spectrometric analyses, a modified silver staining method (Shevchenko et al., 1996) including reduction with dithiothreitol and reaction with iodoacetamide, avoiding glutaraldehyde and minimizing formaldehyde contact, was used.

Sample preparation for mass spectrometry. Protein bands of interest were excised from the silver-stained gel, as was a region of the gel without visible protein, which served as a concurrent control. Samples were destained and subjected to in-gel digestion with trypsin overnight at $37^{\circ} \mathrm{C}$ (UCSF, 2002). The resulting digest solutions were transferred to fresh Eppendorf tubes and $5 \mu \mathrm{L}$ of $1: 1$ water-acetonitrile containing $5 \%$ formic acid was added. The remaining gel pieces were treated with $50 \mu$ of $1: 1$ water-acetonitrile containing $5 \%$ formic acid, mixed for $10 \mathrm{~min}$, centrifuged at $12,000 \times \mathrm{g}$, sonicated for a further $5 \mathrm{~min}$ and the resulting supernatants were transferred to fresh Eppendorf tubes. The samples were vortexed, centrifuged at $12,000 \times \mathrm{g}$ and dried completely under vacuum.

Sample preparation for MALDI-TOF-MS was carried out as previously described (Sze et al., 1998). Briefly, samples were suspended in $30 \mu \mathrm{l}$ of $0.1 \%$ aqueous trifluoroacetic acid (TFA). C18 zip-tips were used to enrich the samples and remove salts and other contaminants. Samples were then eluted twice in $3 \mu$ of $9: 1$ acetonitrile-water containing $0.1 \%$ (v/v) TFA directly onto Au-plated or stainless steel MALDI targets. A 1:1 acetonitrile-water containing $0.1 \%$ ( $/ v$ ) TFA solution saturated with $\alpha$-cyano-4-hydroxycinnamic acid ( $\mathrm{CHCA}, 1 \mu \mathrm{l})$ was mixed with the eluted samples on the target and the spots were air-dried. MALDI-TOF-MS and MALDI-TOF/TOF-MS/MS spectra were collected between $\mathrm{m} / \mathrm{z} 800$ and 5000 using Ciphergen 
PBSII SELDI-TOF or Applied Biosystems 4700 TOF/TOF mass spectrometers in positive ion detection mode. Protein identification was performed via database searches. The Prowl search engine (ProFound version 4.10.5. ProteoMetrics) was used with the following parameters: constrain species to rodent; molecular weight from 20 to $50 \mathrm{kDa}$; mass accuracy $300 \mathrm{ppm}$; use of average mass; trypsin missed cleavages of $0-2$; cysteine modified by iodoacetamide. Following identification of the protein with a statistically significant $Z$ score (Altschul et al., 1997), the protein sequence was retrieved from SwissProt (www.srs.ebi.ac.uk). A list of all tryptic peptides was generated and compared to the original spectra. Internal calibration was carried out using two peptide peaks and the calculated masses from the list. Thereafter, the database search was repeated, resulting in a much higher matching score and greater coverage. For LPMC samples (see below), samples were re-suspended in 9:1 acetonitrile-water containing $0.1 \%$ TFA, bound to $\mathrm{C} 18$ zip-tips, washed with water containing $0.1 \%$ TFA and eluted from the tips with 9:1 acetonitrile-water containing $0.1 \%$ TFA into a concentrated solution of sinnapinic acid in the same solvent. The samples were spotted onto a stainless steel MALDI target and allowed to dry. Samples were then analyzed by MALDI-TOF-MS on an Applied Biosystems 4700 Proteomics Analyzer in linear mode. Porcine DAAO was used as a standard. As an internal control, tryptic peptide samples derived from SDS-PAGE were re-analyzed in CHCA by MALDI-TOF-MS and TOF/TOF-MS/MS on the ABI 4700 instrument in the reflector mode and results were analyzed with SwissProt database searches using

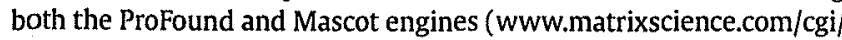
search_form.pl?FORMVER=2\&SEARCH=PMF).

Sample preparation for nanoLC-ESI-QIT-MS analysis consisted of taking the lyophilized tryptic peptides extracted from the gel plugs up into $10 \mu \mathrm{l}$ of $1 \%$ aqueous formic acid with sonication and heating. Aliquots $(1 \mu \mathrm{l})$ were injected. LCMS experiments were performed on a Surveyor nanoflow HPLC system interfaced with a quadrupolar ion trap mass spectrometer (Thermo Finnigan LCQ DECA XP Plus). Data were acquired in positive ion detection mode with the following settings: range of $\mathrm{m} / \mathrm{z} 400-2000$ mass range; precursor charge state set to auto; peptide error tolerance $1.5 \mathrm{Da}$; fragment error tolerance $0.8 \mathrm{Da}$; one missed cleavage; variable modifications of cysteine by carbamidomethylation and of methionine monooxygenation. Data were analyzed by comparison to the keyword "rat" subset of the Swissprot/Uniprot database with Sequest and BioWorks software.

DAAO western blotting and immunohistochemistry in kidney sections. Standard western blotting (Vieira et al., 1994) was carried out with a 1:400 dilution of the primary rabbit anti-porcine-DAAO IgG (provided by Dr. B. Curti, Department of Physiology and Biochemistry, University of Milan, Italy) for $2 \mathrm{~h}$ at room temperature. Kidney sections were deparaffinized and reactivated by immersion in TPBS ( $2 \times 10 \mathrm{~min}$ ), followed by immersion in $7.6 \mathrm{mM}$ citrate buffer, $\mathrm{pH} 6.0$, and microwaving $(3 \times 1 \mathrm{~min}, 700 \mathrm{~W})$. Sections were then rinsed with PBS ( $2 \times 5 \mathrm{~min})$ and non-specific binding was blocked with normal goat serum (20 min, 1:500 in PBS). Sections were incubated for $1 \mathrm{~h}$ with the primary antibody (sheep anti-porcine DAAO IgG, Biotrend, Germany, diluted $1: 1000$ in $1 \%$ BSA-PBS) at room temperature, followed by PBS washing $(2 \times 5 \mathrm{~min})$, and incubation with the secondary anti-sheep IgG-AP (Sigma-Aldrich, 1:50 in 1\% BSA-PBS) for $30 \mathrm{~min}$ at room temperature. Endogenous alkaline phosphatase was blocked with $5 \mathrm{mM}$ levamisol for $10 \mathrm{~min}$. DAAO-specific immunopositive staining was visualized with Fast Red $(8 \mathrm{~min})$. Sections were counter-stained with hematoxylin $(7 \mathrm{~min})$ and covered with aqueous mounting medium (Crystal Mount, Biomeda Corp., USA) and cover slips were applied.

Laser microdissection and pressure catapulting (LMPC). For laserassisted microdissection and pressure catapulting (Emmert-Buck et al., 1996), a PALM LMPC system (PALM Microlaser GmbH, Germany) was used. Areas of interest (whole cortex or individual nuclei) were dissected from $H \& E$ stained whole kidney sections and stored at $-80^{\circ} \mathrm{C}$ until required. Samples were de-waxed with xylene and processed for subsequent MS analysis.

\section{Results}

\section{Animal weights and food intake}

Although propiverine-treated animals of both sexes were lighter (unpaired $t$-test: $p<0.05$ ) than their respective controls (see supplemental information, Figure S2 A and B), which was consistent with a lower food intake per cage, no differences from standard weight-gain tables (www.criver.com) for $\mathrm{F} 344$ rats could be discerned during the course of the study for any of the control or treatment groups. At the end of the study all animals had a body weight considered normal for F344 rats of this age. Animal behavior was normal throughout the course of the study.

\section{Pathological analysis of HEE stained renal sections}

Renal sections from control male F344 rats (Figs. 2A and B) showed the typical cortical and medullary changes associated with $\alpha 2 u$ nephropathy (Swenberg et al., 1989): hyaline droplets in the renal cortical tubules (Fig. 2A), tubular dilatation, cell shedding, protein casts in the cortico-medullary junction (Fig. 2B), tubular regeneration, early signs of chronic progressive nephropathy and occasional glomerular nephropathy retrograde to tubules overburdened with hyaline droplets. In contrast, renal sections from female control rats, presented with no pathology whatsoever (data not shown).

Treatment of male rats with $1000 \mathrm{ppm}$ propiverine for 16 weeks led to an accumulation of hyaline droplets in the cytosol and nuclei of proximal tubule epithelial cells (Fig. 2C), mildly exacerbated cell shedding and exfoliation, proteinaceous casts at the cortico-medullary junction, and mild regenerative proliferation. Female F344 rats treated for 16 weeks with $1000 \mathrm{ppm}$ propiverine also presented with an accumulation of hyaline droplets in the cytosol and nuclei of the proximal tubule epithelial cells (Fig. 2D). However, although this cytosolic and nuclear hyaline droplet accumulation was comparable to the accumulation observed in the corresponding propiverine-treated male rats, no other pathological changes were observed. Propiverinetreated female F344 rats thus differed in the observed renal pathology from the corresponding female control rats only in the accumulation of hyaline droplets in the cytosol and nuclei of proximal tubule epithelial cells.

\section{Gel electrophoresis of nuclear and cytosolic proteins}

SDS-PAGE analyses of proteins from liver nuclei extracts did not reveal any differences in the proteins from control and propiverinetreated rats of either sex (Fig. $3 \mathrm{E}$ ). In contrast, the analysis of renal cortical epithelial cell nuclear proteins demonstrated a protein band that was more prominently expressed in propiverine-treated rats of either sex than in the corresponding controls (Fig. 3A). Based on three independent experiments, molecular weight (MW) calculations provided a MW $39.7 \pm 1.5 \mathrm{kDa}$ for this protein.

\section{Identification of the upregulated protein via MS and databank mining}

Analysis of this $39.7 \mathrm{kDa}$ protein by mass spectrometry (MALDITOF-MS) resulted in spectra clearly distinct from the nuclear protein background. Submission of the tryptic peptide masses found for the nuclear protein isolated from renal cortical epithelial cells of propiverine-treated male and female F344 rats to various database searches (e.g., ProFound Prowl search engine (Zhang and Chait, 2000)), along with the molecular weight calculated via SDS-PAGE 

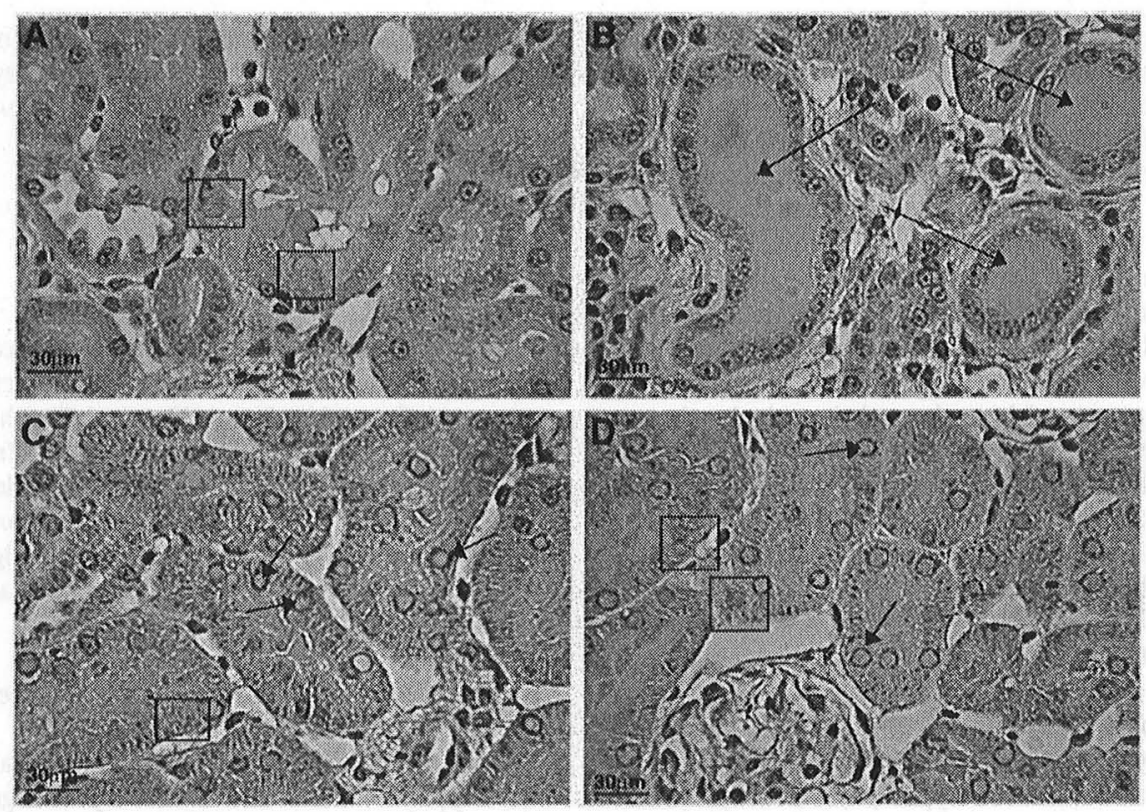

Fig. 2. Histologic findings of hematoxylin-eosin stained kidney sections of male and female F344 rats treated for 16 weeks with 1000 ppm propiverine. A, and B are from a male control rat, C and D, from a propiverine-exposed male and female rat, respectively. A: hyaline droplets (boxes) in the cytosol of a proximal tubule; B: proteinaceaous casts (arrows) at the cortico-medullary junction; C: hyaline droplet accumulation in the nuclei (arrows) and the cytosol (boxes) of proximal tubular cells of a propiverine-exposed male rat; D: protein accumulation in the nuclei (arrows) and the cytosol (boxes) of proximal tubular cells of a propiverine-exposed female rat.

analysis allowed unambiguous identification of this protein as rat $\mathrm{D}-$ amino acid oxidase (DAAO; see Table 1).

\section{Protein verification via western blot}

The presence or absence of DAAO in the protein band observed in the nuclear and cytosolic protein preparations of the renal cortex of propiverine-exposed and control rats (Figs. $3 \mathrm{~A}$ and $\mathrm{C}$ ) was confirmed via western blot analysis with specific antibodies against DAAO (Fig. 3B and D). Faint DAAO expression was detected in renal nuclei extracts from control male rats, while extracts from control female rats yielded no clear DAAO positive reaction, i.e., suggesting absence of DAAO in the nuclei of control females. A pronounced increase in the expression of DAAO was observed in nuclei extracts of propiverineexposed rats of both sexes. Generally, DAAO expression in propiverine-exposed rats was several-fold that of the corresponding controls (Fig. 3B), when using the two corresponding 2 and $20 \mu \mathrm{g}$ purified DAAO controls as a means of comparison. In contrast DAAO was detectable in the renal cytosol of male and female control F344 rats, albeit the level of expression appeared lower in female rats (Fig. 3D). Propiverine-treatment increased DAAO expression in male and female rats when compared to the corresponding control rats and the 2 and $20 \mu \mathrm{g}$ purified DAAO controls. Although no densitometric analyses were carried out, a comparison of the level of staining in the western blots of renal nuclei (Fig. 3B) and cytosolic (Fig. 3D) extracts suggests the level of renal nuclear DAAO expression to be far higher than cytosolic. In contrast, DAAO could not be detected in liver nuclei or cytosolic extracts from control or propiverine-treated rats (Figs. 3E-H).

Confirmation of protein identity and localization via mass spectrometric analysis of laser microdissection and pressure catapulting (LPMC) samples

LMPC was used to excise 3210 nuclei containing excessive protein and corresponding whole renal cortex from H\&E-stained renal sections (Emmert-Buck et al., 1996) of a propiverine-exposed male rat, as well as the prominent SDS-PAGE protein bands of male and female propiverine-exposed rats (Table 2). The samples were pooled and subjected to both MALDI-TOF-MS and nanoLC-ESI-QIT-MS analyses (Zhang et al., 2003) with subsequent protein database comparison. These analyses reconfirmed DAAO as the protein accumulating in the cytosol and nuclei of renal cortical epithelial cells of male and female F344 rats following exposure to propiverine.

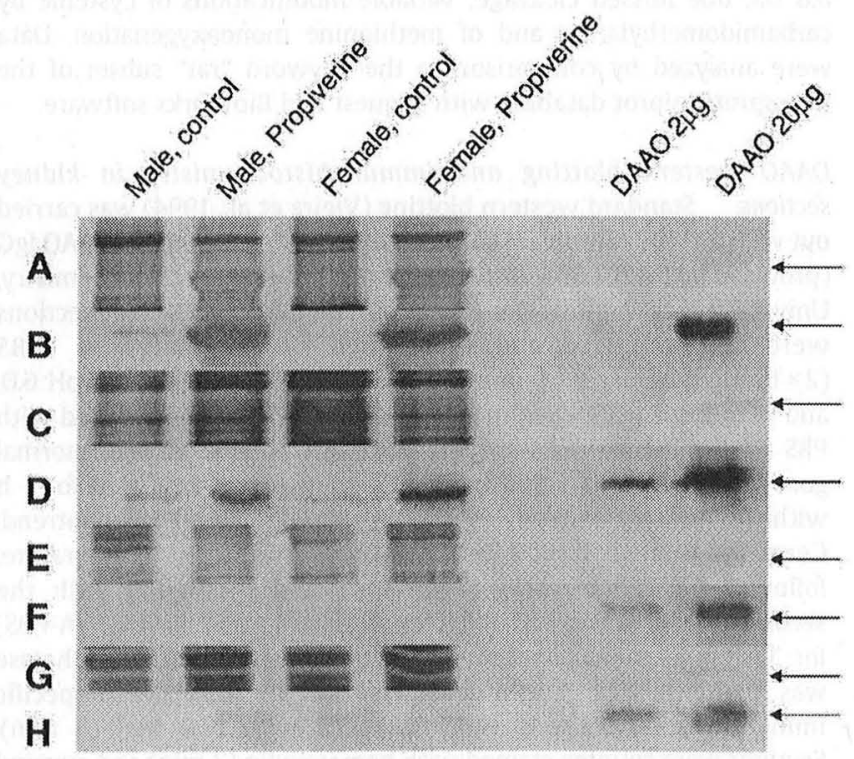

Fig. 3. SDS-PAGE and western (DAAO-immuno)-blot analysis of nuclear and cytosolic proteins. A: Silver-stained SDS-PAGE of nuclear renal proteins; B: DAAO-immunoblotting of nuclear proteins; C: Silver-stained SDS-PAGE of cytosolic renal proteins; D: DAAO-immunoblotting of cytosolic proteins; E: silver-stained SDS-PAGE of nuclear liver proteins; F: DAAO immunoblotting of nuclear liver proteins; G: silver-stained SDS-PAGE of cytosolic liver proteins; $\mathrm{H}$ : DAAO immunoblotting of cytosolic liver proteins; Arrows: protein of interest (approximately $39 \mathrm{kDa}$ ). 
Table 1

profound Search results achieved using the tryptic peptide masses generated with MALDI-TOF analyses and the molecular weight of the protein band identified in the rat renal proximal epithelial cell nuclei following propiverine treatment

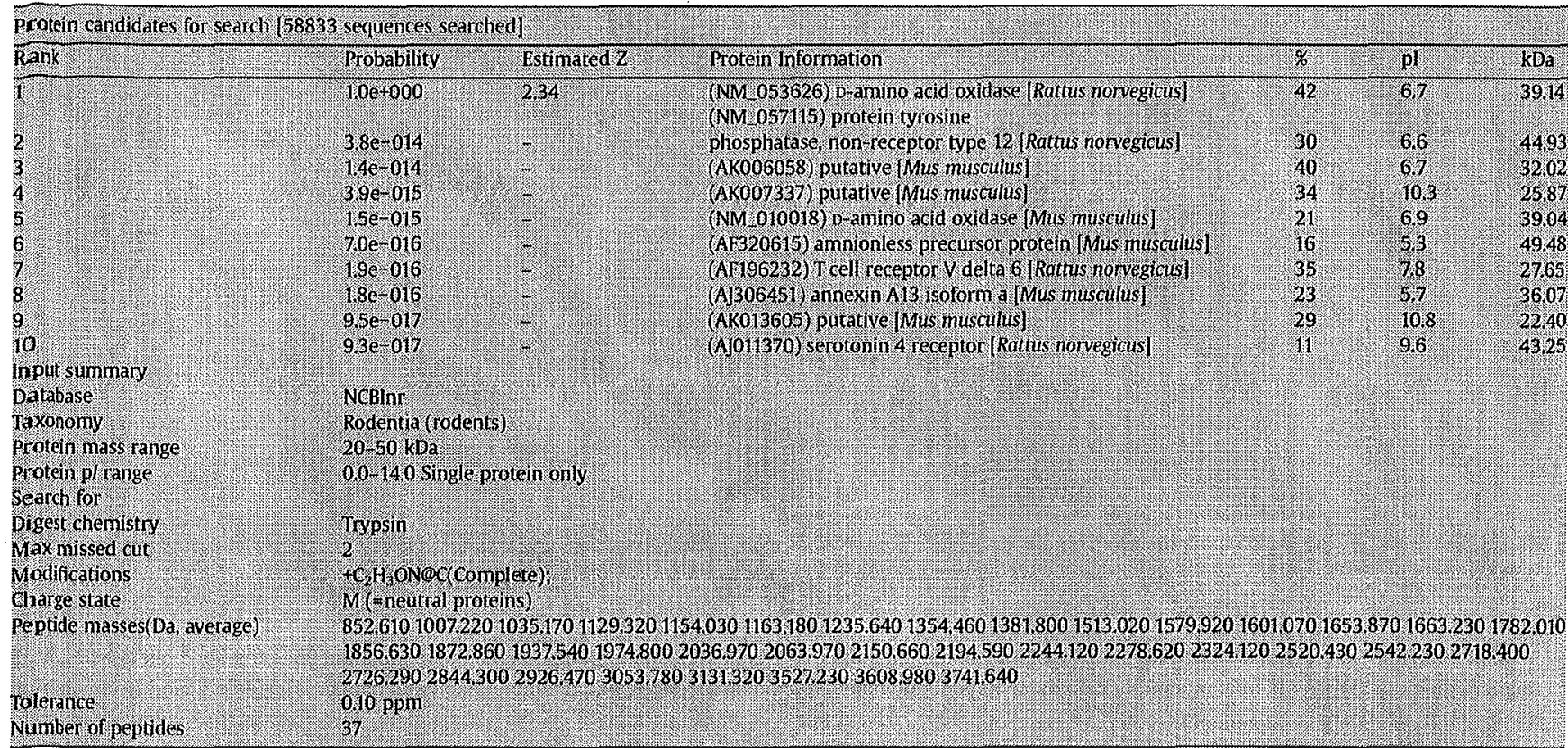

The MS/MS spectrum of the $\mathrm{N}$-terminal peptide from DAAO found in propiverine-exposed male rats is shown in Fig. 4.

\section{Protein verification via immunohistochemistry}

Control male rat renal sections were negative for DAAO immunostaining (Fig. 5A), whereas sections from propiverine-exposed male F344 rats demonstrated DAAO immunopositive staining in the nuclei and cytosol of proximal tubule epithelial cells (segments S2 and S3), Fig. 5B).

\section{Discussion}

In the previous 52-week and the 2-year rat carcinogenicity study (Inoue et al., 1989a) as well as in the study presented here, male and female F344 rats treated with propiverine presented with nuclear and cytosolic protein accumulations in the renal cortex, but not the renal medulla, papilla or in hepatocytes of the liver. A single protein responsible for the observed hyaline droplet accumulations (Fig. 2) was identified with mass spectrometry (Tables 1 and 2 and Fig. 4), and confirmed by western blotting (Fig. 3) and immunohistochemistry (Fig. 5) to be D-amino acid oxidase (DAAO). DAAO is a highly conserved enzyme inherently expressed in the kidneys of rats (Zaar et al., 1986) mice (Koibuchi et al., 1995), pigs and humans (D'Aniello et al., 1993). DAAO is not only expressed in the cytosol (particularly peroxisomes) of the kidney and liver (Angermüller and Fahimi, 1988; D'Aniello et al. 1993) but also in the astrocytes and neurons of the rat and mouse brain (Moreno et al., 1999; Shoji et al., 2006), catalyzing the oxidative deamination and chiral inversion of d-amino acids (Xin et al., 2005) Generally, the expression of DAAO is much higher in rat renal peroxisomes than in liver peroxisomes (Zaar et al., 1986). Protein comparisons for sequence and structural homology demonstrated that mouse DAAO has the highest homology (94\%) to rat (Fig. 6). Human DAAO lacks, amongst others, the 25th and 27 th amino acid residues found in rat, mouse and hamster and thus has only $81 \%$ similarity and $90 \%$ identity to rat DAAO (Konno, 1998; Konno, 2001).

Table 2

Summary of MALDI-TOF-MS and MALDI-TOF/TOF-MS/MS re-analyses of samples for confirmation of DAAO as the accumulating protein in the nuclei of the rat renal proximal tubule epithelial cells following propiverine treatment

\begin{tabular}{|c|c|c|}
\hline mpl & Sam & Results: \\
\hline & 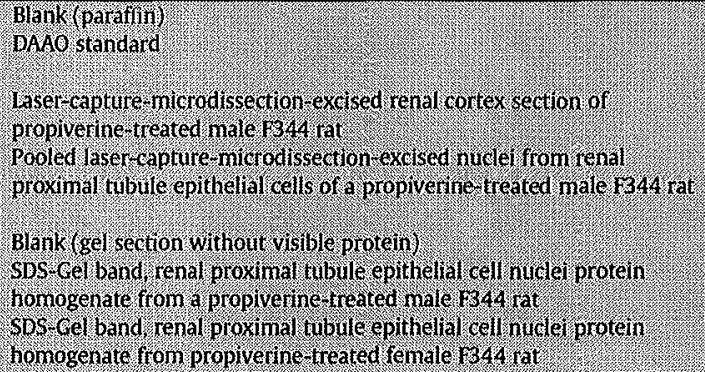 & 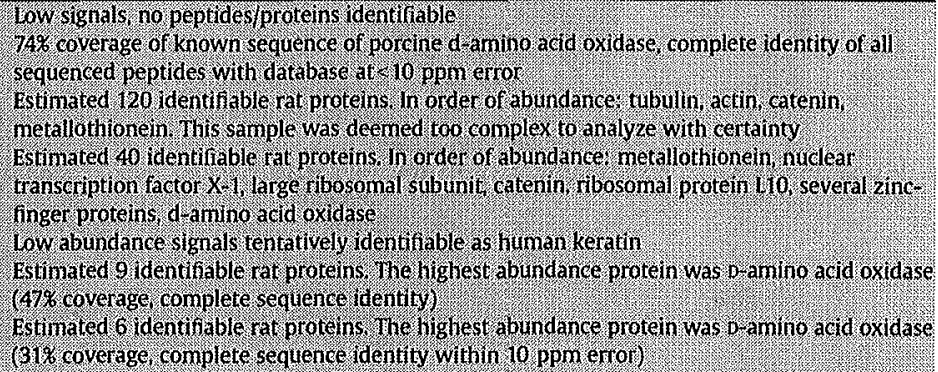 \\
\hline
\end{tabular}

Notes. The 47\% (sample 6) vs. 31\% (sample 7) coverage between samples " 6 " and "7", along with relative signal intensities, is suggestive of relative abundance, i.e., sample 6 appeared to contain more D-amino acid oxidase than did sample 7. 


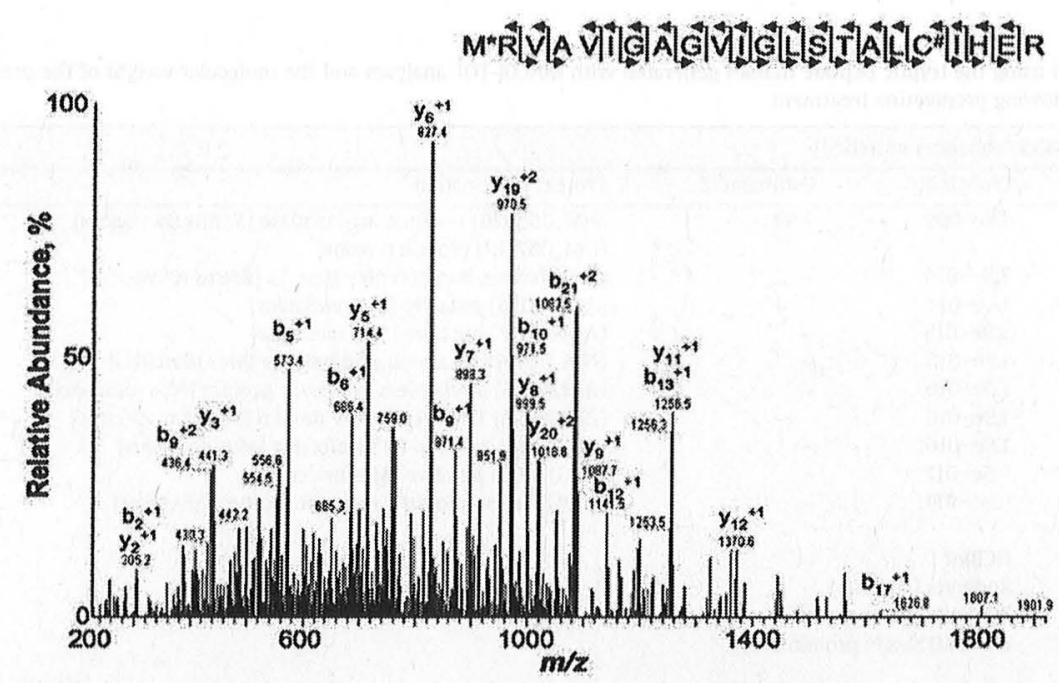

Fig. 4. Quadrupolar ion trap MS/MS spectrum of the $[\mathrm{M}+3 \mathrm{H}] 3+\mathrm{ion}(\mathrm{m} / \mathrm{z} 780.9)$ of the 22-amino acid $\mathrm{N}$-terminal tryptic peptide from kidney nuclei of propiverine-exposed male rats. A nearly replete series of $b$ and $y$ ions was obtained, a few of which are designated on the spectrum. *: Monooxygenated. \#: Carbamidomethylated.

Consistent with the peroxisomal localization, the DAAO amino acid sequences (Fig. 6) of all four species (rat, mouse, pig and human) contain a microbody targeting sequence (Keller et al., 1991). However contrary to earlier findings in rat kidney peroxisomes (Zaar et al., 1986), DAAO expression was not only seen in the peroxisomes of the S2 and S3 segments of the proximal tubule, but was also present in the nuclei of the epithelial cells of the same segments. A finding confirmed via immunohistochemistry (Fig. 5B), western blot (Fig. 3) and mass spectrometric analyses (Table 1 and 2, Fig. 4). The detection of DAAO also in nuclei of the S2 and S3 segments of control male rats (Fig. 3B), suggests an important albeit yet unknown biological function of this enzyme in the nuclei of male rats, albeit the level of DAAO in nuclei of female rats may have been to low for detection with western blot. However, despite confirmation of DAAO in renal proximal tubule cell nuclei, amino acid sequence comparison (Fig. 6) does not demonstrate the presence of a classical bipartite nuclear localizing signal consensus sequence (Kalderon et al., 1984; Makkerh et al., 1996; Grundt et al., 2007) in any of the species compared. DAAO in the renal proximal tubule epithelial cell nucleus, thus may result via co-transport with a specific nuclear protein from the cytosol into the nucleus (Grundt et al., 2007) and could be involved in the metabolism of long-chain fatty acids in the nucleus proper.

Despite that female rats exposed to $1000 \mathrm{ppm}$ propiverine had a marginally lower food intake (see supplemental information, Figure S2 B), female rats had a slightly higher overall oral exposure to propiverine (on average $66.7 \mathrm{mg} \mathrm{kg}^{-1} \mathrm{bw} \mathrm{day}^{-1}$ ) than their corres- ponding male counterparts (on average $58.2 \mathrm{mg} \mathrm{kg}^{-1}$ bw day ${ }^{-1}$ ), when assuming equal bioavailability of propiverine from food in female and male rats. Not surprisingly, the ensuing pathological analysis of kidneys of propiverine-exposed female rats revealed the same level of DAAO accumulation in the cytosol and nuclei of proximal tubule epithelial cells (see Fig. 2D) as that observed in the corresponding exposed male F344 rats (Fig. 2 C). The latter thus suggests that the marginal difference in propiverine exposure had no discernible effect on the development and intensity of DAAO accumulation in the kidneys.

The increased expression of DAAO in the nuclei, and to a lesser extent in the cytosol of the renal proximal tubule epithelial cells of propiverine-treated male and female rats, raises two important questions: a) does increased DAAO expression represent a potential health risk via the production of excessive hydrogen peroxide? and $b$ ) what is the mechanism of this propiverine-mediated increase in DAAO expression? DAAO catalyzes the oxidative deamination of D-amino acids, thereby producing 2-oxo-acids, ammonia and hydrogen peroxide (Krebs, 1935) which, at high enough concentrations, are known cytotoxicants. d-Serine has been demonstrated to be a good substrate for DAAO (Dixon and Kleppe, 1965) and is also nephrotoxic in rats (Williams et al., 2005). d-Serine, however, is not nephrotoxic in DAAO-deficient LEA/SENDAI rats (Maekawa et al., 2005), and its nephrotoxicity can be dose-dependently attenuated in F344 rats with the DAAO inhibitor sodium benzoate (Williams and Lock, 2005; Xin et al., 2005). The latter data suggest that in the absence of D-serine,
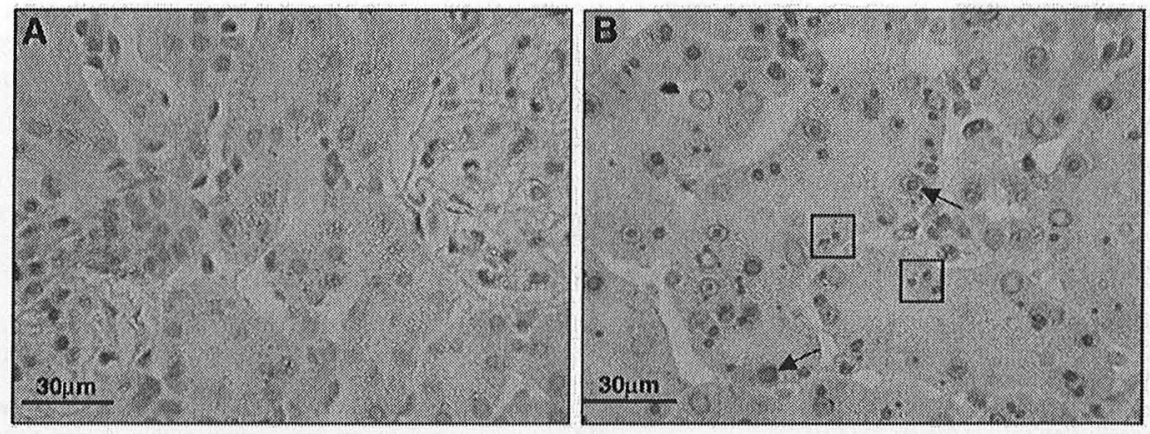

Fig. 5. DAAO immunostaining of kidney sections from a control male rat (A) and a $1000 \mathrm{ppm}$ propiverine-exposed male rat (B). Note intensive positive staining (red) of protein droplets in the nuclei (arrows) and the cytosol (boxes). 


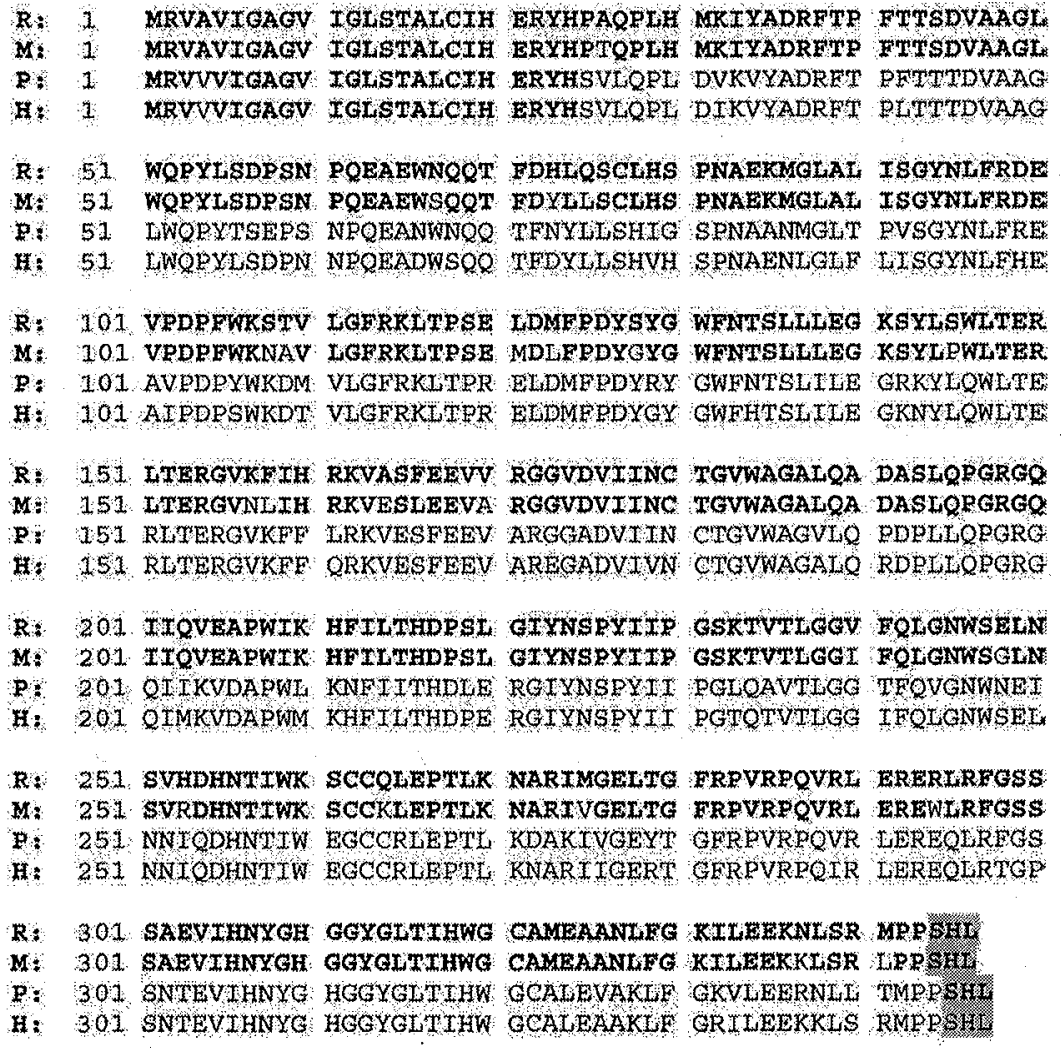

\section{Microbody targeting signal, SHL}

Fig. 6. DAAO amino acid sequences of rat $(\mathrm{R})$, mouse (M), pig $(\mathrm{P})$ and human $(\mathrm{H})$, as derived from SwissProt. Bold letters describe sequence identities between rat and mouse DAAO. Microbody targeting signal, SHL (shaded grey).

DAAO-mediated D-amino acid or fatty acid metabolism in the kidneys of rats will not result in excessive 2-oxo-acids, ammonia and hydrogen peroxide levels and thus cytotoxicity. Indeed, this interpretation is strongly corroborated by the fact that the 16 -week propiverine exposure-induced pathology in F344 rats is exclusively restricted to an accumulation of protein (DAAO) in the nuclei and the cytosol of the renal cortex proximal tubules (S2 and S3) of both sexes. All other pathological changes, observed in male rats only, appear to be associated with the inherent male rat-specific $\alpha 2 \mathrm{u}$-globulin nephropathy (Swenberg et al., 1989), and chronic progressive nephropathy observed also in aging control rats. Thus, no pathological lesions in the liver or kidney, beyond the excessive renal proximal accumulation of cytosolic and nuclear DAAO, were observed in either male or female F344 rats following propiverine-treatment. Moreover, the observation that the rat DAAO is distinct from the DAAO of other species (Fig. 6), including the human (Konno, 1998; Tsuchiya et al., 2003; Williams and Lock, 2005), may explain the lack of d-serine-induced nephrotoxicity in guinea pigs, rabbits, mice, dogs, hamsters, gerbils (Maekawa et al. 2005) and most likely also in humans. This interpretation is further corroborated by the fact that $\mathrm{d}$-serine is normally found in mouse plasma (Nagata et al., 1992) without concurrent nephrotoxicity, despite the presence of active DAAO in the kidney (Koibuchi et al. 1995). The latter data thus support a rat-specific DAAO-mediated mechanism of d-serine nephrotoxicity, and, in the absence of d-serine, no excessive formation of the cytotoxic 2-oxo-acids, ammonia and hydrogen peroxide and therefore lack of renal pathology. This was also confirmed by the absence of propiverine/DAAO-accumulation-specific renal pathology in male and female rats following 16 weeks (this study) and 104 weeks propiverine-treatment (Inoue et al., 1989a), beyond the accumulation of DAAO in the cytosol and nuclei of proximal tubule epithelial cells. Therefore, the present data from rodents and other species, suggest that despite the presence of $d$ serine in human blood, urine and the brain (Bruckner and Hausch, 1993; Hashimoto et al., 1993; Bruckner et al., 1994), human DAAO, even in the unlikely event of being excessively expressed in patients following chronic propiverine treatment, would not produce excessive amounts of 2-oxo-acids, ammonia or hydrogen peroxide. Thus humans (specifically patients treated with propiverine) would not experience renal or neuronal toxicity and indeed no reports of such toxicity exist despite the use of propiverine in patients since 1981 in Germany and later in other European countries and Japan.

The question remains, however, as to the mechanism by which propiverine induces the observed overexpression of DAAO in the renal proximal nuclei and cytosol of rats. The absence of propiverineinduced DAAO accumulation in the kidneys of mice, despite 94\% DAAO amino acid sequence identity, and beagle dogs is suggestive of a ratspecific mechanism underlying the DAAO accumulation phenomenon.

One of the best characterized species-specific mechanisms is the hypolipidemia- and phthalate-induced increased peroxisome proliferation and peroxisomal-enzyme induction in the livers of rats and mice (Peters et al., 1996). The mechanism of peroxisome proliferation, enzyme induction and ensuing pathological changes observed in the liver, and occasionally the kidneys of exposed rats and mice, is apparently mediated through the peroxisome proliferator activated receptor $\alpha$ (PPAR $\alpha$ ) and is largely dependent on the strength of the agonist's interaction with PPAR $\alpha$ (Peters et al., 1996). The analysis of PPAR $\alpha$ agonist-induced effects in livers and kidneys of rats and mice, although rather diverse, reveals some resemblance to the biochemical/ 
pathological changes observed in propiverine-treated rats as reported here. Indeed, increased DAAO expression and activity has been found in kidneys of lactating rats and their pups exposed to ciprofibrate, whereas the liver levels of DAAO are significantly lower than in the corresponding controls (Stefanini et al., 1999). The suppression of DAAO in the liver of PPAR $\alpha$ agonist (phthalates, clofibrate)-treated rats (Lake et al., 1984; Reubsaet et al., 1991) appears to be a general phenomenon and compares well with the absence of DAAO induction (Fig. 3) and lack of peroxisome proliferation in the livers of propiverine-treated rats (data not shown, (Inoue et al., 1989a)), while concurrently an increased DAAO activity was observed in the kidneys (Reubsaet et al., 1991). Moreover, treatment of male and female F344 rats with the hypolipidemic RMI 14,514 (5-tetradecyloxy-2-furancarboxylic acid) lead to a marked change in peroxisome morphology and an increased activity of three hydrogen peroxide producing oxidases (including DAAO) in the liver of both sexes, but only to a slight increase in DAAO activity in the kidney. The latter observations suggest that DAAO expression in the kidneys of rats, as is that of many other mitochondrial, peroxisomal and microsomal enzymes involved in the process of $\beta$-oxidation of long-chain fatty acids in the renal proximal tubule, is under the control of PPAR $\alpha$. Indeed, clofibrate treatment of rats led to a marked increase in liver and kidney PPAR $\alpha$ expression (Bartosiewicz et al., 2001). PPAR $\alpha$ is the predominantly expressed PPAR in rat renal proximal tubule epithelial cells (Yang et al., 1999) and is causally related to the clofibrate-induced expression of P-4504A1 (Ishizuka et al., 2003), an enzyme intricately involved in fatty acid oxidation in the rat (Tollet et al., 1994). Moreover, PPAR $\alpha$ regulates amino acid metabolism (Kersten et al., 2001) most likely via regulation of DAAO expression in the kidney. All of the above strongly suggests that DAAO expression in the renal cortex of rats is associated with fatty acid metabolism, possibly mediated via a PPAR $\alpha$-regulated pathway. The mechanism by which propiverine mediates an increased expression of DAAO in the peroxisomes and nuclei of the rat renal proximal tubule epithelial cells remains to be investigated. Preliminary in vivo and in vitro data however do support an interaction of propiverine and propiverine-N-oxide (Fig. 1) with the rat PPAR $\alpha$ (Heussner et al., 2008) but not with human PPARs (Dietrich, unpublished data).

In view of the absence of pathological effects in the liver and kidneys, including absence of DAAO accumulation, of mice and beagle dogs chronically treated with propiverine and in addition to the fact that PPAR $\alpha$ agonist-associated diseases in the rat, including liver, pancreatic acinar cell and testicular Leydig cell tumors, have been found to be rat-specific and thus not relevant to humans (Gonzales, 1997; Klaunig et al., 2003; Gonzalez and Shah, 2008), the observed DAAO accumulation in the rat kidneys following chronic propiverineexposure may also be a rat-specific effect, not readily extrapolated to other species, including the human.

\section{Acknowledgments}

The authors gratefully acknowledge the technical assistance of $P$. Wolf and A. Prietz with DAAO immunohistochemistry and animals, respectively, and Dr. Emanuel M. Schreiber for the LC-MS analyses.

\section{Appendix A. Supplementary data}

Supplementary data associated with this article can be found, in the online version, at doi:10.1016/j.taap.2008.09.014.

\section{References}

Altschul, S.F, Madden, T.L., Schäffer, A.A., Zhang, J., Zhang, Z., Miller, W., Lipman, D.J. 1997. Gapped BLAST and PSI-BLAST: a new generation of protein database search programs. Nucleic Acid Res. 25, 3389-3402.

Angermüller, S., Fahimi, H.D., 1988. Heterogenous staining of $\mathrm{D}$-amino-acid oxidase in peroxisomes of rat liver and kidney. Histochemistry 88, 277-285.

Bartosiewicz, M.J., Jenkins, D., Penn, S., Emery, J., Buckpitt, A., 2001. Unique gene expression patterns in liver and kidney associated with exposure to chemical toxicants. J. Pharmacol. Exp. Ther. 297, 895-905

Blobel, G., Potter, R.V., 1966. Nuclei from rat liver: isolation method that combines purity with high yield. Science 154, 1662-1665.

Bradford, M., 1976. A rapid and sensitive method for the quantitation of microgram quantities of protein utilizing the principle of protein-dye binding. Anal, Biochem. 72, 248-254

Bruckner, H., Hausch, M., 1993. Gas chromatographic characterization of free D-amino acids in the blood serum of patients with renal disorders and of healthy volunteers. J. Chromatogr. 614, 7-17.

Bruckner, H., Haasmann, S., Friedrich, A., 1994. Quantification of D-amino acids in human urine using GC-MS and HPLC. Amino Acids 6, 205-211.

D'Aniello, A, D'Onofrio, G., Pischetola, M., D'Aniello, $G$, Vetere, A.., Petrucelli, L, Fisher G.H., 1993. Biological role of D-amino acid oxidase and D-aspartate oxidase. Effects of D-amino acids. J. Biol. Chem. 268, 26941-26949.

Dietrich, D.R., 1995. Alpha 2u-globulin: Species- and sex-specific protein synthesis and excretion, association with chemically induced renal toxicity and neoplasia in the male rat and relevance in human cancer risk assessment. In: Hodgson, E., Bend, $J . R$ Philpot, R.M. (Eds.), Reviews in Biochemical Toxicology. Toxicology Communications Inc, Raleigh, North Carolina, USA, pp. 115-180.

Dietrich, D.R., Rasonyi, T., 1995. Preneoplastic lesions in kidney and carcinogenesis by non-genotoxic compounds. In: Degen, G.H., Seiler, J.P., Bentley, P. (Eds.), Toxicology in Transition, Springer, Berlin.

Dietrich, D.R., Swenberg, J.A., 1991a. Alpha2u-globulin is necessary for D-limonene promotion of male rat kidney tumors. Toxicologist 11, 1296

Dietrich, D.R., Swenberg, J.A. 1991b. NCl-Black-Reiter (NBR) male rats fail to develop renal disease following exposure to agents that induce $\alpha 2 u$-globulin $\left(\alpha_{2 u}\right)$ nephropathy. Fundam. Appl. Toxicol. 16, 749-762.

Dietrich, D.R., Swenberg, J.A., 1991c. The presence of $\alpha_{2 u}$-globulin is necessary for Dlimonene promotion of male rat kidney tumors. Can. Res, 51, 3512-3521.

Dixon, M., Kleppe, K., 1965. D-Amino acid oxidase II. Specificity, competitive inhibition and reaction sequence. Biochim. Biophys. Acta 96, 368-382.

EMEA 2003. Phasing-in EU procedures: MRP and referrals. Pan European Regulatory Forum, London, www.hma,eu/uploads/media/PERF_MRP_DCP. Phasing in_01.pdf, p. 9.

Emmert-Buck, M.R., Bonner, R.F., Smith, P.D., Chuaqui, R.F., Zhuang, Z, Goldstein, S.R. Weiss, R.A., Liotta, L.A., 1996. Laser capture microdissection. Science 274, 998-1001 Gonzales, FJ., 1997. Recent update on the PPARa-null mouse. Biochimie 79, 139-144.

Gonzalez, FJ, Shah, YM, 2008. PPARalpha: mechanism of species differences and hepatocarcinogenesis of peroxisome proliferators. Toxicology $246,2-8$.

Gopinath, C., Prentice, D.E., Lewis, D.j., 1988. Atlas of experimental toxicologic pathology. MTP Press, Lancaster, UK.

Grundt, K., Haga, I.V., Huitfeldt, H.S., Ostvold, A.C., 2007. Identification and characterization of two putative nuclear localization signals (NLS) in the DNA-binding protein NUCKS, Biochim. Biophys. Acta 1773, 1398-1406.

Hashimoto, A., Kumashiro, S. Nishikawa, T., Oka, T., Takahashi, K., Mito, T., Takashima, S, Doi, N., Mizutani, Y., Yamazaki, T., et al., 1993. Embryonic development and postnatal changes in free $\mathrm{D}$-aspartate and $\mathrm{D}$-serine in the human prefrontal cortex. J. Neurochem, 61, 348-351.

Heukeshoven, J., Dernik, R., 1985. Simplified method for silver staining of proteins in polyacrylamide gels and the mechanism of silver staining. Electrophoresis 6 , 103-112.

Heussner, A.H., Baldock, M.l, Braeter, M.l, Dietrich, D.R., 2008, Characterization of propiverine effects on rat PPARs. Toxicologist 92 (S-1), 93.

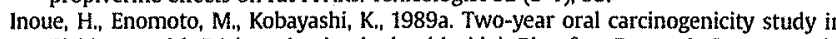
F344 rats with P4 (propiverine hydrochloride). Biosafety Research Center, Foods Drugs and Pesticides (An-Pyo-Center), Shizuoka, Japan, Report No. 1556 (Apogepha ID: P 148), pp. 267.

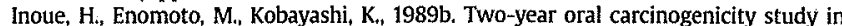
mice (B6C3F1) with P4 (propiverine hydrochloride). Biosafety Research Center Foods, Drugs and Pesticides (An-Pyo-Center), Shizuoka, Japan, Report No. 1557 (Apogepha ID: P 150), p. 258.

Ishizuka, T., Ito, O., Tan, L., Ogawa, S., Kohzuki, M., Omata, K., Takeuchi, K., Ito, S., 2003. Regulation of cytochrome $\mathrm{P}-4504 \mathrm{~A}$ activity by peroxisome proliferator-activated receptors in the rat kidney. Hypertens. Res. 26, 929-936,

Kalderon, D., Roberts, B.L, Richardson, W.D., Smith, A.E., 1984, A short amino acid sequence able to specify nuclear location. Cell 39, 499-509.

Keller, G.A., Krisans, S., Gould, S.j., Sommer, J.M., Wang, C.C., Schliebs, W., Kunau, W. Brody, S., Subramani, S., 1991. Evolutionary conservation of a microbody targeting signal that targets proteins to peroxisomes, glyoxysomes, and glycosomes. J. Cell Biol, 114, 893-904.

Kersten, S., Mandard, S., Escher, P., Gonzalez, F.J., Tafuri, S., Desvergne, B., Wahli, W. 2001. The peroxisome proliferator-activated receptor alpha regulates amino acid metabolism. FASEB J. 15, 1971-1978.

Klaunig, J.E., Babich, M.A., Baetcke, K.P., Cook, J.C., Corton, J.C., David, R.M., DeLuca, J.G., Lai, D.Y, Mckee, R.H., Peters, J.M., Roberts, R.A., Fenner-Crisp, P.A., 2003. PPARalpha agonist-induced rodent tumors; modes of action and human relevance. Crit. Rev. Toxicol, 33, 655-780.

Kohda, S., Nishikawa, H., Sumino, M., Sano, M., Kuroda, J., Miura, K., Tsuchitani, M. Narama, I., Yada, H., Yamashita, K., 1989. One-year chronic oral toxicity study of propiverine hydrochloride in dogs followed by one-month recovery. J. Tox. Sci. 14, $111-160$.

Koibuchi, N., Konno, R., Matsuzaki, S., Ohtake, H., Niwa, A., Yamaoka, S., 1995. Localization of $\mathrm{D}$-amino acid oxidase mRNA in the mouse kidney and the effect of testosterone treatment. Histochem. Cell Biol. 104, 349-355.

Konno, R, 1998. Rat D-amino-acid-oxidase cDNA: rat D-amino-acid-oxidase as an 
intermediate form between mouse and other mammalian $\mathrm{D}$-amino-acid-oxidases. Biochim. Biophys. Acta 1395, 165-170.

Konno, R., 2001. Assignement of D-amino acid oxidase gene to a human and a mouse chromosome. Amino Acids 20, 401-408

Krebs, H.A., 1935. Metabolism of amino-acids: deamination of amino-acids. Biochem. J. 29. 1620-1644.

Laemmli, U.K., 1970. Cleavage of structural proteins during the assembly of the head of bacteriophage T4. Nature 227, 680-685.

Lake, B.G., Rijcken, W.R., Gray, T.J., Foster, J.R., Gangolli, S.D., 1984. Comparative studies of the hepatic effects of di- and mono-n-octyl phthalates, di-(2-ethylhexyl) phthalate and clofibrate in the rat. Acta Pharmacol. Toxicol. (Copenh) 54, 167-176.

Madersbacher, H., 2006. Orale Anticholinergika bei überaktiver Blase. Urologe 45, 830-834.

Madersbacher, H., Mürtz, G., 2001. Efficacy, tolerability and safety profile of propiverine in the treatment of the overreactive bladder (non-neurogenic and neurogenic). World J. Urol. 19, 324-335.

Maekawa, M., Okamura, T., Kasai, N., Hori, Y., Summer, K.H., Konno, R., 2005. D-aminoacid oxidase is involved in D-serine-induced nephrotoxicity. Chem. Res. Toxicol. 18, 1678-1682.

Makkerh, J.P., Dingwall, C., Laskey, R.A., 1996. Comparative mutagenesis of nuclear localization signals reveals the importance of neutral and acidic amino acids. Curr Biol. 6, 1025-1027.

May, K., Westphal, K., Giessmann, T., Wegner, D., Adam, U., Lerch, M.M., Oertel, R., Warzok, R.W., Weitschies, W., Braeter, M., Siegmund, W., 2008. Disposition and antimuscarinic effects of the urinary bladder spasmolytics propiverine; influence of dosage forms and circadian-time rhythms. J. Clin. Pharmacol. 48, 570-579.

May, K., Giessmann, T. Wegner, D., Oertel, R., Modess, C., Oswald, S., Braeter, M. Siegmund, $W_{1}$ in press. Oral absorption of propiverine solution and of the immediate and extended release dosage forms: influence of regioselective intestinal elimination. Eur, J. Clin. Pharmacol, doi:10.1007/s00228-008-0528-0.

Moreno, S., Nardacci, R., Cimini, A., Ceru, M.P., 1999. Immunocytochemical localization of D-amino acid oxidase in rat brain. J. Neurocytol. 28, 169-185.

Nagata, Y., Yamamoto, K., Shimojo, T., Konno, R. Yasumura, Y., Akino, T., 1992. The presence of free D-alanine, D-proline and D-serine in mice. Biochim. Biophys. Acta $1115,208-211$.

Peters, J.M., Zhou, Y.C, Ram, P.A., Lee, S.S., Gonzales, F.., Waxman, D.J., 1996. Peroxisome proliferator-activated receptor alpha required for gene induction by dehydroepiandrosterone-3-beta-sulfate. Mol. Pharmacol. 50, 67-74.

Reubsaet, F.A., Veerkamp, J.H., Bruckwilder, M.L, Trijbels, J.M., Monnens, L.A., 1991 Peroxisomal oxidases and catalase in liver and kidney homogenates of normal and di(ethylhexyl)phthalate-fed rats. Int. J. Biochem. 23, 961-967.

Shevchenko, A., Wilm, M., Vorm, O., Mann, M., 1996. Mass spectrometric sequencing of proteins from silver-stained polyacrylamide gels. Anal. Chem. 68, 850-858.

Shoji, K., Mariotto, S., Ciampa, A.R., Suzuki, H., 2006. Mutual regulation between serine and nitric oxide metabolism in human glioblastoma cells. Neurosci. Lett. 394 163-167.

Stefanini, S., Nardacci, R., Farioli-Vecchioli, S., Pajalunga, D., Sartori, C., 1999. Liver and kidney peroxisomes in lactating rats and their pups after treatment with ciprofibrate. Biochemical and morphometric analysis. Cell. Mol. Biol. (Noisy-le-grand) 45, 815-829.

Swenberg, J.A, 1991, Risk assessment of chemicals causing alpha2u-globulin nephropathy. Reg. Toxicol. Pharmacol. 13, 1-2.

Swenberg, J.A., Short, B., Borghoff, S., Strasser, J. Charbonneau, M., 1989. The comparative pathobiology of alpha 2u-globulin nephropathy. Toxicol. Appl. Pharmacol. 97, 35-46.

Sze, E.T., Chan, T.W., Wang, G., 1998. Formulation of matrix solutions for use in matrixassisted laser desorption/ionization of biomolecules. J. Am. Soc. Mass Spectrom. 9, $166-174$.

Tollet, P., Stromstedt, M., Froyland, L, Berge, R.K., Gustafsson, J.A., 1994. Pretranslational regulation of cytochrome P4504A1 by free fatty acids in primary cultures of rat hepatocytes, J. Lipid Res, 35, 248-254.

Tsuchiya, M., Kurabayashi, A., Konno, R., 2003. Hamster D-amino-acid oxidase cDNA: rodents lack the 27 th amino acid residue in D-amino-acid oxidase. Amino Acids 24 , 223-226.

UCSF, 2002. University of California San Francisco In-gel digest procedure. http:/I donatello.ucsf.edu/ingel.html.

Vieira, A., Elkin, R., Kuchler, K., 1994. Western blotting and ligand blotting using enhanced chemiluminescence and radioiodine detection. In: Celis, J.E. (Ed,), Cell Biology, A Laboratory Handbook. Academic Press Inc., London, pp. 314-321.

Williams, R.E., Lock, E.A., 2005. Sodium benzoate attenuates D-serine induced nephrotoxicity in the rat. Toxicology 207, 35-48.

Williams, R.E., Major, H., Lock, E.A., Lenz, E.M., Wilson, I.D, 2005. D-Serine-induced nephrotoxicity: a HPLC-TOF/MS-based metabonomics approach. Toxicology 207, 179-190.

Wuest, M., Hecht, J., Christ, T., Braeter, M., Schoeberl, C., Hakenberg, O.W., Wirth, M.P., Ravens, U., 2005. Pharmacodynamics of propiverine and three of its main metabolites on detrusor contraction. British J. Pharmacol. 145, 608-619.

Xin, Y.F., Zhou, X.J., Cheng, $X$, Wang, Y.X. 2005. Renal D-amino acid oxidase mediates chiral inversion of $N(G)$-nitro-D-arginine. J. Pharmacol. Exp. Ther. 312, 1090-1096.

Yamashita, K., Kuwata, M., Irimura, K., Morinaga, H., Kurokawa, K., Ashizawa, M., 1990. Fifty-two weeks oral chronic toxicity study of propiverine hydrochloride in rats. J. Tox. Sci. 15, 107-144.

Yang, T., Michele, D.E, Park, J., Smart, A.M., Lin, Z, Brosius Ill, F.C., Schnermann, J.B., Briggs, J.P., 1999. Expression of peroxisomal proliferator-activated receptors and retinoid $X$ receptors in the kidney. Am. J. Physiol. 277, F966-973.

Zaar, K., Angermuller, S. Volk1, A., Fahimi, H.D., 1986. Pipecolic acid is oxidized by renal and hepatic peroxisomes. Implications for Zellweger's cerebro-hepato-renal syndrome (CHRS). Exp. Cell Res. 164, 267-271.

Zhang, W., Chait, B.T. 2000. ProFound: an expert system for protein identification using mass spectrometric peptide mapping information. Anal. Chem. 72, 2482-2489.

Zhang, W., Krutchinsky, A.N., Chait, B.T., 2003. "De novo" peptide sequencing by MALDIquadrupole-ion trap mass spectrometry: a preliminary study. J. Am. Soc. Mass Spectrom. 14, 1012-1021. 